\section{Escales \\ y Pont de Suert aprovechamientos hidroeléctricos del Noguera Ribagorzana}

Breve reseña descriptiva de dos de los aprovechamientos hidroeléctricos de la cuenca del río Noguera Ribagorzana, es decir, la presa de Escales y el salto do Pont de Suert. Los estudios, proyectos y trabajos do estas obras han sido realizados por la Empresa Nacional Hidroeléctrica del Ribagorzana.

El río Noguera Ribagorzana, afluente del Segre, sirve de divisoria entre las provincias de Lérida y Huesca en su curso superior; su cuenca se alimenta, principalmente, del deshielo de nieves de alta montaña. La cabecera de este río se halla enclavada en la zona de lagos y cubetas naturales de gran altitud, particularidad que da al Ribagorzana características propias de todos los ríos de montaña, y por tanto, favorables a los aprovechamientos hidroeléctricos.

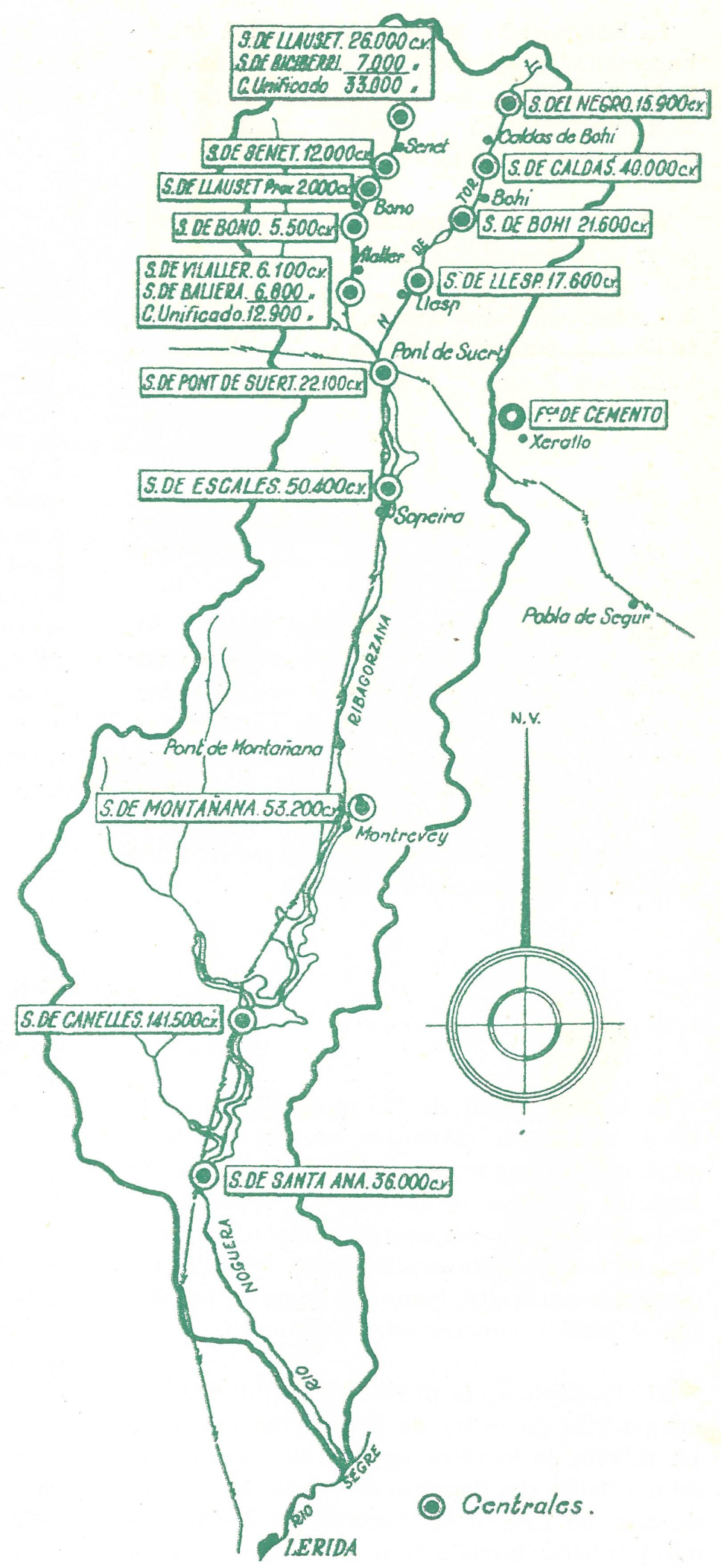

INSTITUTO TECNICO DE LA CONSTRUCCION Y DEL CEMENTO 
La Empresa Nacional Hidroeléctrica del Ribagorzana, más conocida por las iniciales E. N. H. E. R., se ha encargado de la explotación hidroeléctrica de esta importante cuenca fluvial pirenaica oriental.

Para el aprovechamiento total de esta cuenca se han proyectado varias obras, algunas de las cuales se hallan en explotación actualmente; otras, en construcción, y el resto, en estudio. Este trabajo se refiere exclusivamente a la presa de Escales y al salto de Pont de Suert.

aprovechamiento hidroeléctrico de Escales

Las obras del salto de Escales, sobre el río Noguera Ribagorzana, están constituídas por una presa de gravedad y una central subterránea que, por sus condiciones particulares, presentan características interesantes. La presa es de tal esbeltez que aventaja, en este sentido, a la mayor parte de las presas de gravedad actualmente existentes. La central subterránea es la más importante en España.

Ia presa

De tipo gravedad, de $125 \mathrm{~m}$ de altura a partir de la base de cimientos, se proyecta horizontalmente según una alineación recta. El embalse de agua se eleva a 157 millones de metros cúbicos, cuya energía almacenada será aprovechada tan favorablemente, que sólo los depósitos naturales, como los lagos de montaña, se pueden superar en rendimiento.

El aliviadero de la presa se ha equipado con compuertas de sector de fabricación nacional. La válvula de toma de agua y las compuertas de maniobra del desagüe de fondo; la tubería de éste, de $2,40 \mathrm{~m}$ de diámetro, y las "virolas de la tubería forzada han sido suministradas por la industria española.
Terminada la obra se procedió a una serie de inyecciones de cemento con objeto de impermeabilizarla en la medida de lo posible.

\section{tubería forzada}

Como la central es subterránea, y la tubería forzada pasa a través de una roca sana y compacta, se ha proyectado dicha canalización, haciendo contribuir el terreno para absorber, parcialmente, la presión desarrollada en el interior de la tubería por la diferencia de nivel hidrostático. A esta finalidad se dió a la chapa un pequeño espesor, forzando el metal a una carga de trabajo que se aproxima a la correspondiente a su límite elástico. Esta condición exigió un gran escrúpulo en las operaciones de relleno del hueco que se dejaba entre las virolas y hastiales del pozo por donde desciende la tubería. El procedimiento seguido en esta última operación es el prepakt, es decir, rellenar primero con grava simplemente e inyectar después una lechada o mortero de cemento.

central subterránea

La central subterránea del salto de Escales se halla enclavada en roca caliza; el volumen excavado para formar el hueco previsto para las instalaciones de los grupos generadores es de gran consideración. En esta serie de aprovechamientos escalonados se han previsto unas cuantas centrales más de este mismo tipo, y hasta de mayor importancia.

En la central se han instalado tres grupos, equipados con turbinas Francis de 16.800 CV cada uno y alternadores de $15.000 \mathrm{~kW}$. Para la transformación se han instalado dos tipos de transformadores: uno para voltajes medios y otros de salida para grandes voltajes.

Merece especial mención el cable empleado en la galería subterránea, con voltaje de 150.000 y provisto de un aislamiento por circulación de aceite, que ha sido fabricado por la casa Pirelli, de Villanueva y Geltrú. 


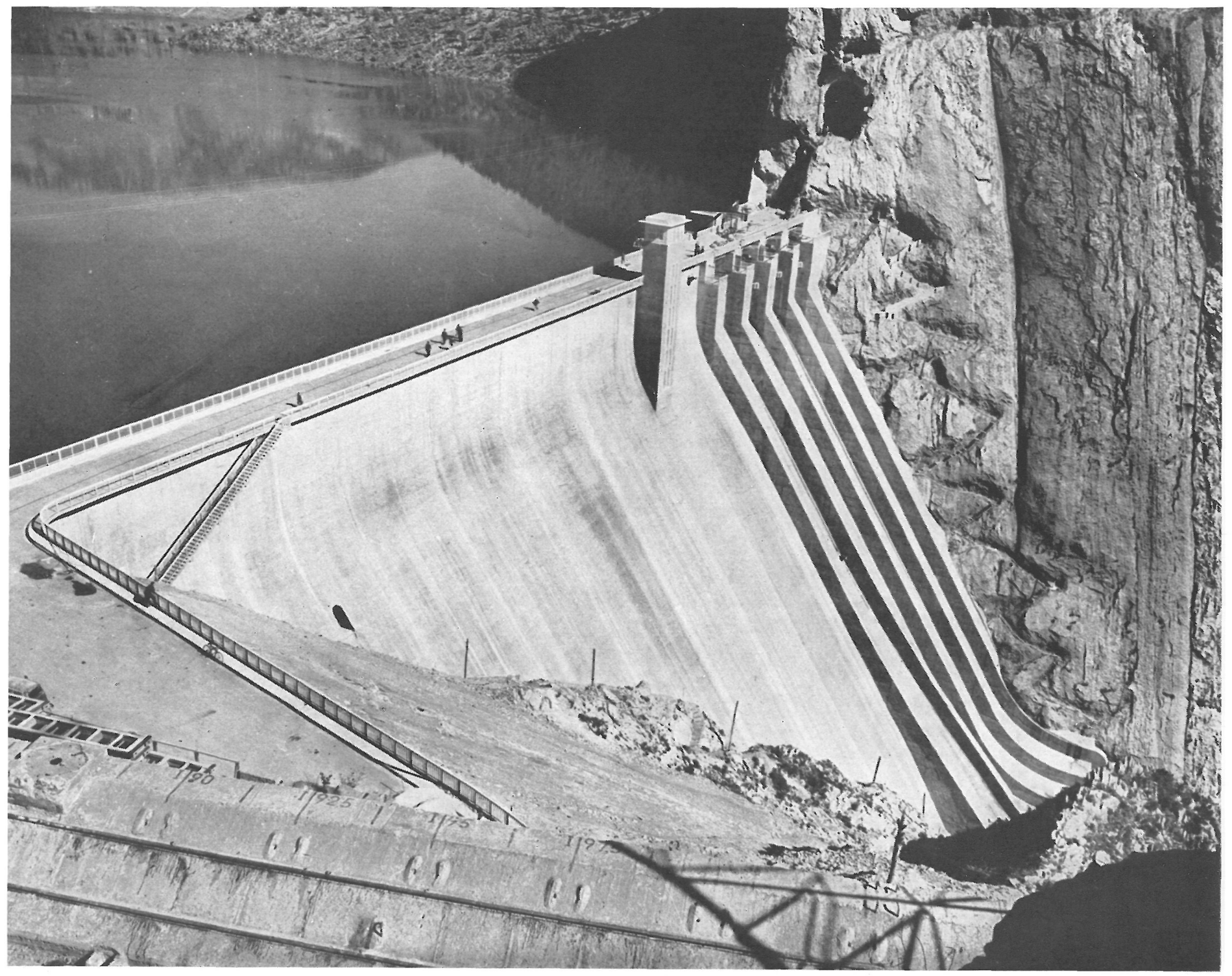

Aspecto general de la presa de Escales, correspondiente a uno de los parte del plan general de explotación de esta cuenca por E. N. H. E. R.

Gran parte del material utilizado en estas instalaciones electromecánicas es de procedencia nacional.

nueva fábrica de cemento

Por presentar el valle del Ribagorzana grandes dificultades de acceso, así como teniendo en cuenta el gran consumo de cemento que todos estos aprovechamientos hidroeléctricos habían de motivar, se procedió a la instalación de una fábrica de cemento en las proximidades de Xerelló. 

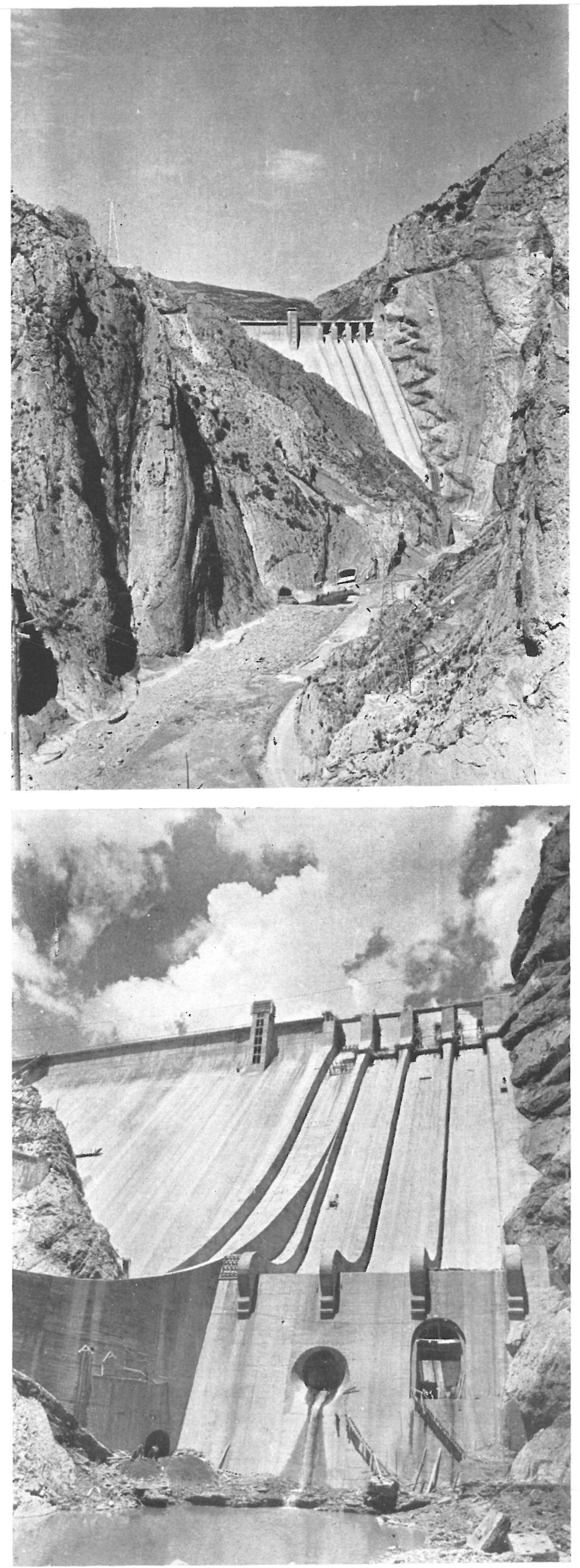

En estas fotografías, tomadas desde aguas abajo de la presa de Escales, se pueden apreciar las condiciones favorables para la cerrada del vaso que ha de constituir el embalse.

La producción inicial, ampliable, de 25.000 t anuales en 1951, alcanzó hasta 63.000 t en 1954.

salto de Pont de Suert

El salto de Pont de Suert es el quinto de una serie similar de aprovechamientos, en los que se turbinan las aguas derivadas de una o varias fuentes de aportación. El salto con derivación de Pont de Suert aprovecha las aguas derivadas del Noguera Ribagorzana, Noguera de Tor y del río Baliera. Así, pues, carece de embalse, y, por tanto, de presa de retención propiamente dicha; pero, contrariamente, dispone de tres tomas de agua diferentes, una para cada río.

Las aguas del Ribagorzana se llevan por un canal de unos $4.000 \mathrm{~m}$ de longitud, de 0,0007 de pendiente $y$ $8,50 \mathrm{~m}^{3} / \mathrm{s}$ de caudal, a confluir con las de otro canal, procedente del río Tor, de aproximadamente $3.500 \mathrm{~m}$ de longitud, que toma sus aguas un poco más abajo del salto de Llesp, tiene igual pendiente que el anterior y cuyo caudal es de $11 \mathrm{~m}^{3} / \mathrm{s}$. Una vez unidos los canales del Ribagorzana y Tor, sus aguas pasan el sifón de Ribagorzana. 


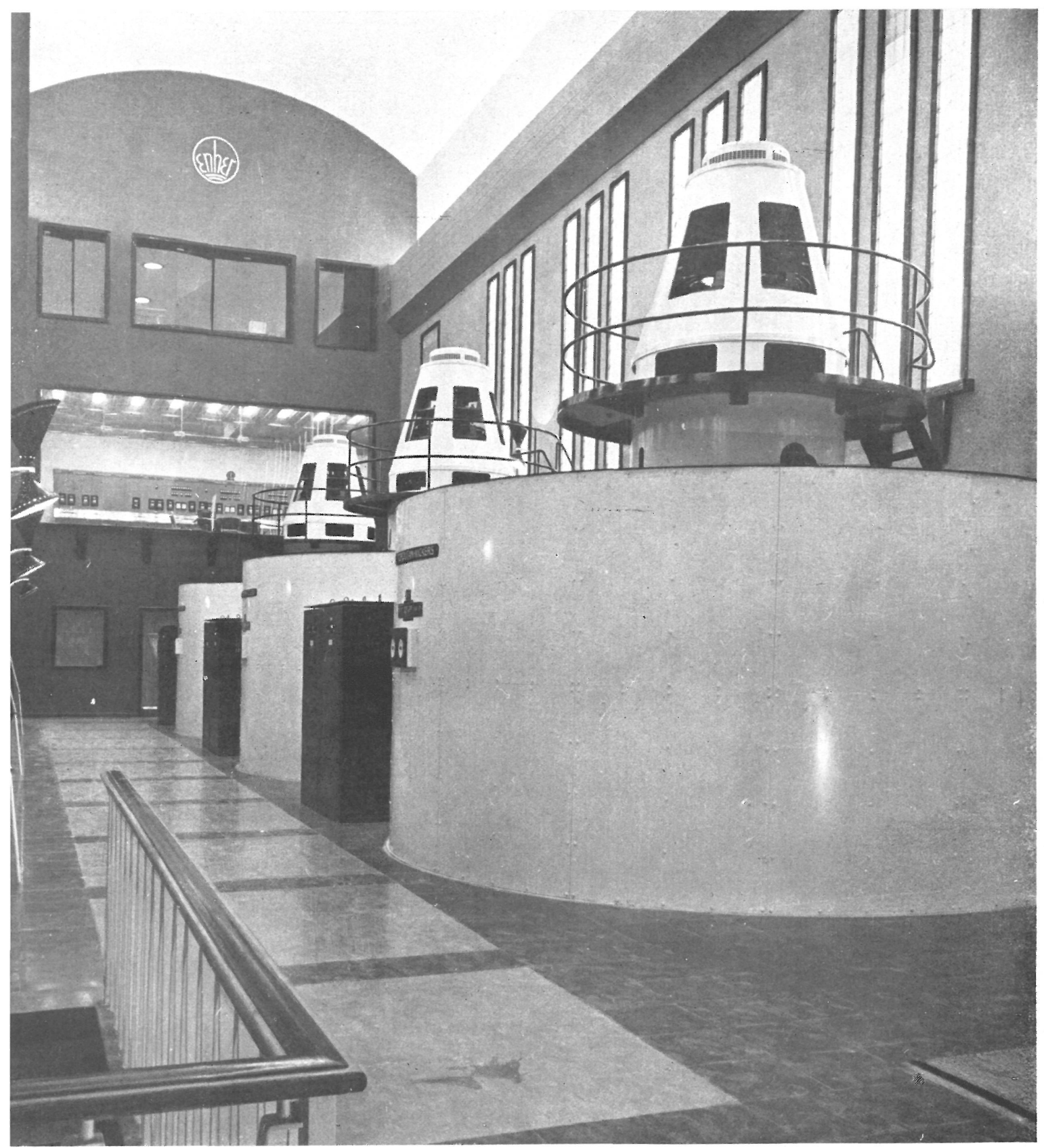

Sala de máquinas de la central subterránea del salto de Escales. Para la formación de la gran cámara que debía alojar la central, se excavaron $30.000 \mathrm{~m}^{3}$ de roca.

A estas aguas se unen las derivadas del río Baliera, conducidas por un tercer canal de unos $3.800 \mathrm{~m}$ de longitud, 0,002 de pendiente y un caudal de $2 \mathrm{~m}^{3} / \mathrm{s}$.

Los caudales reunidos de estos tres canales se conducen a la cámara de carga del salto por medio de un cuarto canal, llamado canal principal, cuya mayor parte del trazado la pasa en galería. Este canal pasa la depresión de Cirés con un sifón metálico de 2,90 m de diámetro; su longitud es de unos $3.000 \mathrm{~m}$; su pendiente, de 0,0006; su caudal, de $21 \mathrm{~m}^{3} / \mathrm{s}$, y desemboca en la cámara de carga de $1.658 \mathrm{~m}^{3}$ de capacidad. 


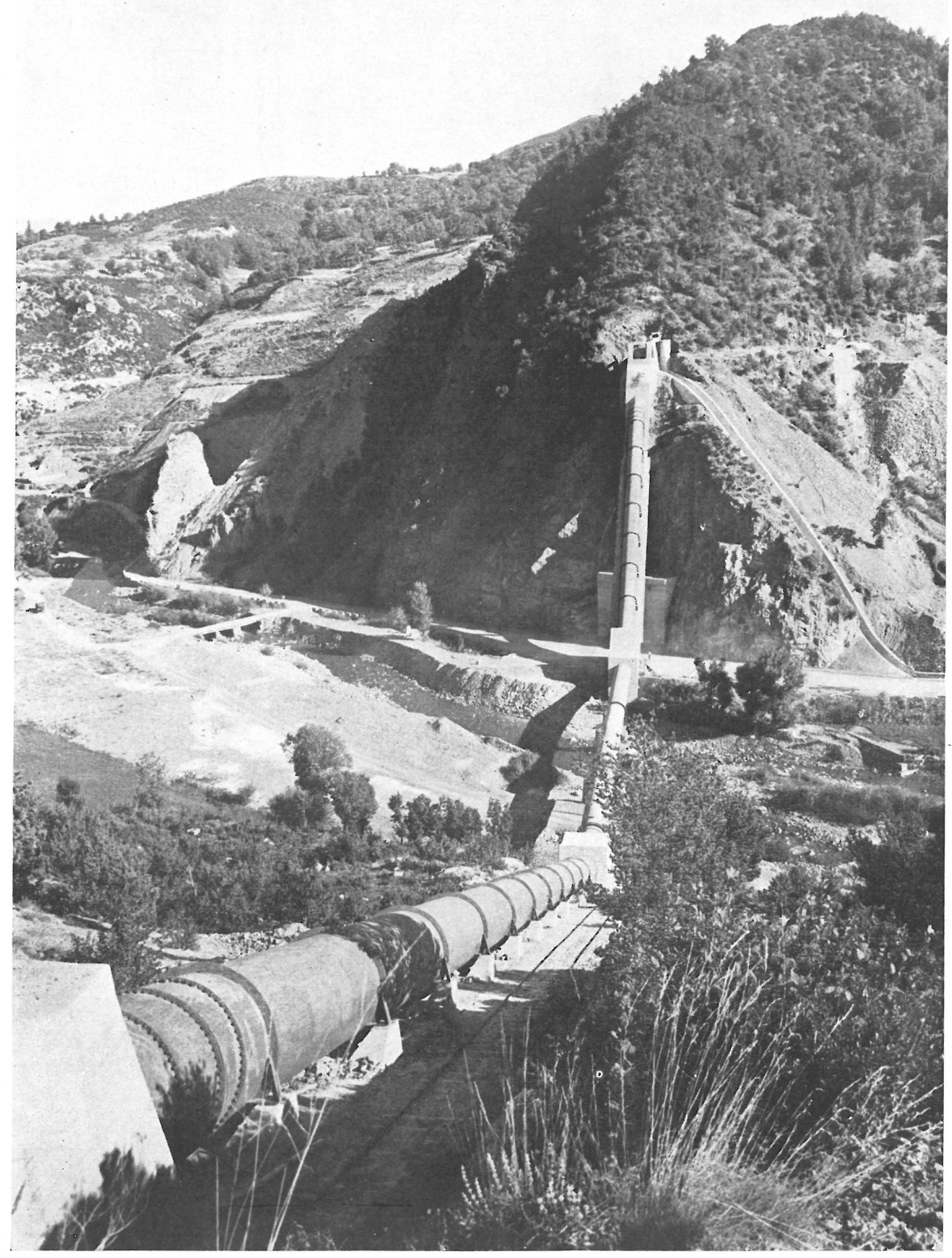

Reunidas las aguas derivadas del Ribagorzana y Tor, pasan la sifón, conocido por el nombre de sifón del mediante

La tubería forzada tiene $135 \mathrm{~m}$ de longitud, 2,50 $\mathrm{m}$ de diámetro y espesor variable de 8 a $21 \mathrm{~mm}$. Tanto esta canalización como las anteriores son de fabricación nacional.

La central de Pont de Suert se ha equipado con dos grupos generadores, cada uno de ellos está formado por una turbina de $10.900 \mathrm{CV}$ de potencia y un alternador de $960 \mathrm{~kW}$. La transformación elevará el voltaje a $132 \mathrm{KV}$.

$$
\text { J. J. U. }
$$

